\title{
Enseñanza de las Ciencias bajo el esquema del proyecto de Ciencia, Tecnología, Ingeniería y Matemáticas (STEM)
}

\author{
Science teaching under the scheme of the Science, Technology, Engineering and Mathematics \\ Project (STEM)
}

OLIVEROS, Maria Amparo†*, VALDEZ, Benjamín, IBARRA, Roberto y ROA, Reyna

Universidad Autónoma de Baja California, Facultad de Pedagogía a e Innovación Educativa, Instituto de Ingeniería

CVU $1^{\text {er }}$ Autor: Maria Amparo, Oliveros / CVU CONACYT ID: 217694

ID 1 ${ }^{\text {er }}$ Coautor: Benjamín, Valdez / CVU CONACYT ID: 13163

ID $2^{\text {do }}$ Coautor: Roberto, Ibarra

ID $3^{\text {er }}$ Coautor: Reyna, Roa / CVU CONACYT ID: 382202

DOI: $10.35429 / J U M .2019 .8 .3 .1 .11$

Recibido 12 de Marzo, 2019; Aceptado 30 de Junio, 2019

\begin{abstract}
Resumen
La industria 4.0 significa la cuarta de una serie de revoluciones industriales, que en su momento transformaron economías, empleos, incluyendo a la sociedad misma, cuya fortaleza deriva de la formación en ciencia y tecnología, sin embargo, la matrícula de estudiantes en carreras de STEM es crítica. Para fortalecer los esfuerzos, en la formación de carreras en STEM se articuló un grupo de colaboración integrado por tres universidades para integrar una red STEM + A (donde A significa la inclusión de las Artes) en la región con el objetivo de que cada vez más jóvenes se inscriban en las áreas de ciencia, tecnología e ingeniería y matemáticas, además de promover la retención y la igualdad de género. En la primera fase, el proyecto se centró principalmente en la medición de indicadores pertinentes utilizando el instrumento IRIS-Q como base de apoyo para el diseño de acciones a implementar en una segunda etapa. Los indicadores resultantes muestran cómo la capacitación cultural, la identidad juvenil y la equidad de género impactan positivamente en las opciones de carreras relacionadas con el STEM por parte de los estudiantes en la zona fronteriza de Baja California y Sonora. Con base a estos resultados se pueden definir cuáles son las estrategias que las universidades deben diseñar e implementar y cuáles son los factores de éxito para reclutar y retener a los estudiantes en las carreras de STEM
\end{abstract}

STEAM, Revoluciones Industriales, Habilidades para el siglo XXI, Educación en ingeniería, Nuevo modelo educativo, Ecosistema STEAM

\begin{abstract}
Industry 4.0 represents the fourth of a series of industrial revolutions, which in turn transformed economies, jobs, including society itself, whose strength derives from training in science and technology, however, the enrollment of students in STEM careers It is critical. To strengthen the efforts, in the formation of careers in STEM, a collaboration group was formed by three universities to integrate a STEM + A network (where A signifies the inclusion of the Arts) in the region with the objective that more and more young people enroll in the areas of science, technology and engineering and mathematics, in addition to promoting retention and gender equality. In the first phase, the project focused mainly on the measurement of relevant indicators using the IRIS-Q instrument as a support base for the design of actions to be implemented in a second stage. The resulting indicators show how cultural training, youth identity and gender equality have a positive impact on career options related to STEM by students in the border area of Baja California and Sonora. Based on these results, you can define which strategies universities should design and implement and what are the success factors for recruiting and retaining students in STEM careers.
\end{abstract}

STEAM, Industrial revolutions, Skills for the 21st century, Engineering education, New educational model, STEAM ecosystem

Citación: OLIVEROS, Maria Amparo, VALDEZ, Benjamín, IBARRA, Roberto y ROA, Reyna. Enseñanza de las Ciencias bajo el esquema del proyecto de Ciencia, Tecnología, Ingeniería y Matemáticas (STEM). Revista de Gestión Universitaria. 2019. 3-8: 1-11

$\dagger$ Investigador contribuyendo como primer autor. 


\section{Introducción}

El modelo STEM de acuerdo a la National Science Foundation (NSF, por sus siglas en inglés 2000) de Estados Unidos, procura capturar el espíritu de la educación, las comunidades y la fuerza de trabajo; que engloban, el pensamiento crítico, el análisis, el trabajo en equipo, en la que los estudiantes integran los procesos y conceptos en el mundo real, para el desarrollo exitoso de competencias para la universidad, el trabajo y la vida.

En la actualidad, el impulso de iniciativas STEM se ha convertido en uno de los objetivos fundamentales de la planificación educativa no sólo de países como Estados Unidos, Reino Unido, Australia, Japón o Finlandia, sino también del conjunto de la Unión Europea y de diversos organismos internacionales. Incluso compañías líderes en diversos sectores, pero en general muy vinculadas al ámbito tecnológico, han unido esfuerzos con las administraciones públicas para desarrollar programas o iniciativas de fomento de las vocaciones tecnológicas entre los jóvenes (Boe, et al 2011: 37-72).

Las revoluciones industriales (Figura 1) han marcado en gran manera la evolución y transformación de la humanidad, llevando al hombre a adaptarse a diversos cambios (Salas, 2016). En la primera Revolución Industrial, entre los siglos XVIII y XIX, se mecanizaron los procesos de producción, transformando la economía agraria y artesanal en otra liderada por la industria (Del Val, 2016). La segunda revolución industrial (1870) implantó las líneas de producción en la planta con reducción en los tiempos de producción y se organiza la producción masiva de productos con características idénticas para su producción por lotes. Y con la generación de la electricidad se diseñan los motores eléctricos como unidades de potencia en los sistemas de manufactura (Carvajal, 2017).

La tercera revolución industrial se basó en las tecnologías de la información y comunicación, así como en las innovaciones que nos permiten el desarrollo de energías renovables. Nunca antes se había logrado el nivel de interactividad o intercomunicación que beneficiara en diferentes niveles a nuestra sociedad, educación e industria (Swain, 2017:12-22).
La cuarta revolución industrial está creando un enfoque centrado en ecosistemas digitales, es decir, está generando modelos de negocios innovadores basados en la interconexión de millones de consumidores, máquinas, productos y servicios (Escudero, 2018: 149-163). Esta revolución se caracteriza por el internet de las cosas, la robótica, la nanotecnología y la inteligencia artificial.
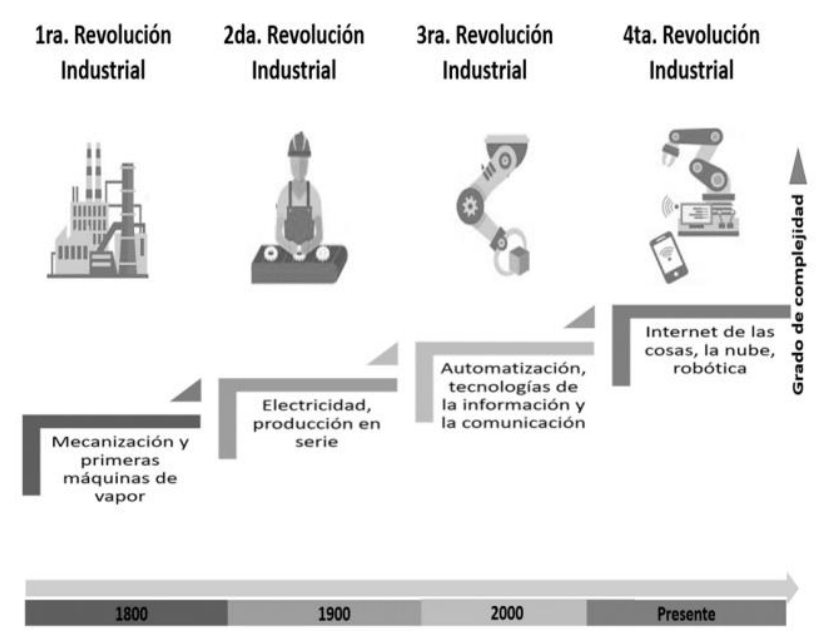

Figura 1 Revoluciones Industrials Fuente: Elaboración Propia

\section{Habilidades necesarias de STEM en la fuerza laboral future}

El desafío de la participación de STEM surge no sólo de la disminución de matrículas en algunas disciplinas, sino también de las necesidades proyectadas de aumentos en la fuerza de trabajo de STEM en el futuro. El informe ampliamente citado, "El mundo necesita más científicos" (UNESCO, 2017), pidió un aumento sustancial del porcentaje de investigadores científicos y tecnológicos en la fuerza de trabajo total. Las proyecciones de varios países indican que la demanda de mano de obra educada en STEM aumentará durante los años venideros y existe una preocupación generalizada de que el suministro de personas educadas dentro de STEM será demasiado pequeño para satisfacer las demandas futuras (Bjørnstad et al., 2008).

De acuerdo al seminario 2017 de UNESCO: "Educar para la $4^{\mathrm{a}}$ revolución industrial", establece que el sistema educativo debe "educar para la $4^{\mathrm{a}}$ revolución industrial" ya que ésta se caracteriza por la fusión de tecnologías emergentes o tecnologías disruptivas, como son los avances en inteligencia artificial, automatización y robótica. 
Los cuales son multiplicados por el gran alcance de conectividad entre miles de millones de personas con dispositivos móviles con acceso sin precedentes a datos y conocimiento.

Además de esto, los avances en la economía digital y la biotecnología, junto con la globalización y el envejecimiento de la población están cambiando la forma en que las personas aprenden, viven y trabajan. Para enfrentar todos estos retos se establecieron dos temas generales: la $4^{\text {a }}$ Revolución Industrial Tendencias, desafíos y oportunidades y Educación y Habilidades para la $4^{\mathrm{a}}$ Revolución Industrial.

La $4^{\mathrm{a}}$ Revolución Industrial Tendencias, desafíos y oportunidades: recomienda de qué manera las empresas y los países deben prepararse para los rápidos cambios del entorno científico y tecnológico. Revisar estrategias e iniciativas para el desarrollo regional, examinar las cadenas de suministro y analizar qué sectores se benefician, ya que todos estos cambios tendrán efecto en la forma en cómo trabajan y viven las personas.

Educación y Habilidades para la $4^{\mathrm{a}}$ Revolución Industrial: la principal preocupación es cómo los países puedan responder y reaccionar a estas tecnologías emergentes. Las principales recomendaciones están en el sentido de disminuir las desigualdades, capacitación a los maestros y alumnos para las nuevas reglas y roles, disminuir la brecha de género. También examinar el papel del sector privado y cuáles son sus contribuciones actuales a la educación y la capacitación y como puede ser su fusión en un futuro. Por ejemplo, cómo pueden colaborar las empresas privadas con el sector educativo para el desarrollo de habilidades que permitan una menor desigualdad. En el caso de los sistemas educativos qué cambios están ocurriendo en el elearning, análisis de aprendizaje, pensamiento crítico, procesos de aprendizaje, entre otros, que den respuesta a las necesidades de la $4^{\text {a }}$ Revolución Industrial.

Habilidades necesarias para la $4^{\mathrm{a}}$ Revolución Industrial

De acuerdo al World Economic Forum el común denominador para tener un empleo en 2015 es tener una profesión ligada a las áreas de ciencia, tecnología, ingeniería y matemáticas (STEM por sus siglas en ingles).
En el cuadro siguiente se hace una muestra comparativa de las habilidades más solicitadas en 2015 y posiblemente en 2025 (Tabla 1).

\begin{tabular}{|c|c|c|}
\hline Habilidades & 2015 & 2025 \\
\hline 1 & $\begin{array}{l}\text { Resolución de } \\
\text { problemas } \\
\text { complejos }\end{array}$ & $\begin{array}{l}\text { Resolución } \\
\text { problemas } \\
\text { complejos }\end{array}$ \\
\hline 2 & $\begin{array}{l}\text { Coordinación con } \\
\text { otros trabajadores }\end{array}$ & Pensamiento crítico \\
\hline 3 & $\begin{array}{l}\text { Juicio y toma de } \\
\text { decisiones }\end{array}$ & $\begin{array}{l}\text { Orientación } \\
\text { servicio }\end{array}$ \\
\hline 4 & Escucha activa & Negociación \\
\hline 5 & Control de calidad & $\begin{array}{l}\text { Inteligencia } \\
\text { emocional }\end{array}$ \\
\hline 6 & Creatividad & Flexibilidad \\
\hline 7 & Negociación & $\begin{array}{l}\text { Coordinación con } \\
\text { otros trabajadores }\end{array}$ \\
\hline 8 & $\begin{array}{l}\text { Orientación } \\
\text { servicio }\end{array}$ & $\begin{array}{l}\text { Juicio y toma de } \\
\text { decisiones }\end{array}$ \\
\hline 9 & Pensamiento crítico & Gestión de personal \\
\hline 10 & Gestión de personal & Creatividad \\
\hline
\end{tabular}

Tabla 1 Habilidades solicitadas por los empleadores Fuente: Elaboración Propia con base en WEF

Según el reporte de la OCDE (2015), México representa el nivel más bajo en los requerimientos del mercado laboral con actividades tecnológicas de alto nivel. Respecto a la OCDE, el $30.9 \%$ de los empleadores mexicanos informan haber enfrentado dificultades para encontrar personas con las competencias necesarias para ocupar vacantes en áreas STEM. Esto se debe a los bajos niveles de innovación en la economía mexicana. Entre los países de la OCDE, México tiene un puntaje muy bajo en materia de patentes y publicaciones científicas relevantes. En relación a la brecha de género, aunque los niños y las niñas tienen destrezas similares en matemáticas y ciencias, su actitud hacia el aprendizaje y las aspiraciones para el futuro son notablemente diferentes, lo cual tiene un efecto importante sobre su decisión de continuar estudios de un nivel más alto y con su elección de carrera (Archer et al, 2012: 881908).

De acuerdo a investigaciones entre las que destacan (Dabney 2013) se ha demostrado que hombres y mujeres, en general poseen capacidades similares para estudiar ciencia y tecnología. La brecha de género en la elección de carreras parece surgir de aspectos actitudinales y de socialización, diferentes en hombres y mujeres. 
De entre los aspectos reportados, se han investigado especialmente la actitud negativa de las mujeres por la ausencia de modelos de mujeres científicas e ingenieras en la educación, la irrelevancia para las mujeres de los temas curriculares, la didáctica y el clima en las clases de ciencia más apropiada para los hombres, la visión masculina de la ciencia y la presión cultural y social de los roles de género sobre las mujeres (Blickenstaff, 2005). Para reducir la brecha de género, es necesario recorrer un largo camino de fomento a la actitud positiva de las niñas hacia el aprendizaje de las matemáticas y de inversión en esta área (Blickenstaff, 2005). De continuar habiendo en esta área tan pocas mujeres que elijan el área STEM, seguirá habiendo pocos modelos que las jóvenes puedan emular y el ciclo simplemente se perpetuará (Scott, 2014).

Las diferencias de género en aspiraciones y expectativas sobre una carrera pueden ser uno de los factores que conducen a un mercado de trabajo segregado por género lo que, a su vez puede tener consecuencias no deseadas para los individuos y las sociedades (Uthoff, 2006). La ausencia de mujeres en el mercado laboral se asocia con un menor desarrollo y crecimiento económico

\section{Los enfoques de aprendizaje en ingeniería y el nuevo modelo educativo SEP}

El nivel de bienestar y la competitividad de las naciones dependen de la cantidad y calidad de sus científicos e ingenieros. Actualmente, en la mayor parte de los países desarrollados hay una preocupación especial por atraer jóvenes hacia esas disciplinas y formarlos con la mayor calidad posible.

La Figura 2, muestra los principales enfoques para mejorar la atracción de la ingeniería y facilitar el aprendizaje desde etapas tempranas hasta la licenciatura. Los retos del siglo XXI para la sociedad del conocimiento implican un cambio de estrategias educativas para impulsar el desarrollo económico del país. El modelo STEM es la integración de la ciencia, tecnología, ingeniería y matemáticas, combinando el aprendizaje en el aula con las experiencias del mundo real (Sanders, 2009), para proporcionar a los estudiantes las competencias profesionales y técnicas con el fin de lograr el desarrollo de la ciencia y la tecnología necesarias para elevar la calidad de vida de la población.

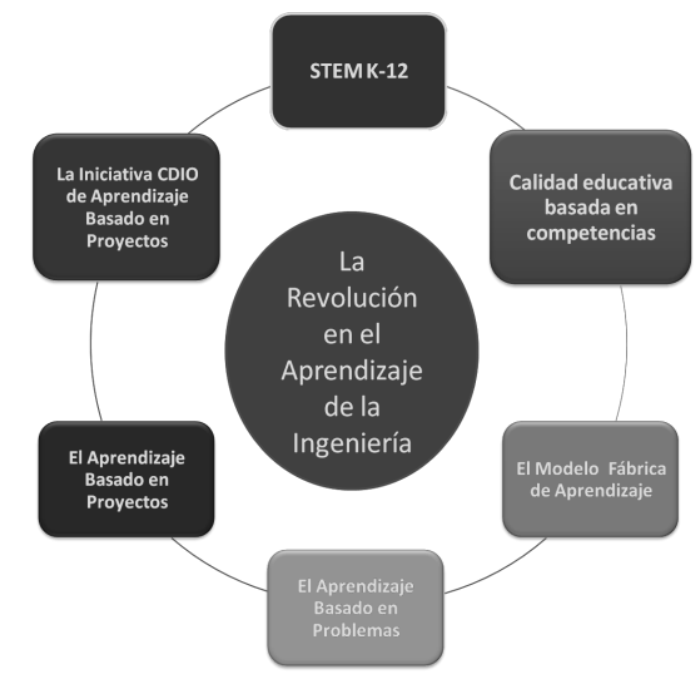

Figura 2 Nuevos enfoques de aprendizaje en ingeniería. Sanders, 2009

\section{El modelo STEM}

De acuerdo a (Toma, 2015) diversos autores, el STEM es un enfoque interdisciplinar cuyos objetivos principales son (a) responder a los desafíos económicos presentes en todas las naciones, (b) identificar las necesidades de los trabajadores que requieren un conocimiento más flexible y nuevas habilidades para ajustarse a los requisitos laborales y sociales actuales, y (c) hacer hincapié en la necesidad de solucionar los problemas tecnológicos y medioambientales a través de la alfabetización científica de los estudiantes. Objetivos que proponen la importante necesidad de la inclusión del STEM en los actuales modelos educativos.

\section{STEM como herramienta para incentivar los estudios en Ciencias e Ingeniería}

Con el objetivo de impulsar la inserción de las mujeres en el sector productivo, a su vez como elemento fundamental para el desarrollo económico, la (OCDE,2015) establece políticas educativas para América Latina basada en el modelo STEM. Dicho modelo combina un número de diferentes tipos de contenido, en el que acentúan las estrategias educativas con el fin de motivar y evitar la deserción de las mujeres en la ingeniería, con estrategias como, 1) Estancias en las empresas, para la aplicación práctica de los conocimientos adquiridos en la universidad, 2) Implementación de talleres vivenciales y lectura crítica, 3) Debates, mesas de discusión y pensamiento crítico, 4) Ferias y concursos de ciencias, clubes y redes de investigación, y 5) Talleres y platicas de mentores exitosos en las áreas STEM, como se puede apreciar en la Figura 3. 


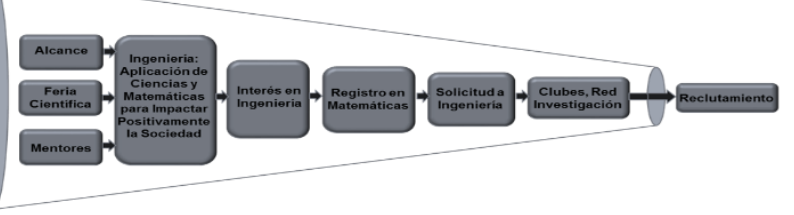

Figura 3 Modelo STEM: Fuente OCDE 2015

\section{Metodología}

El instrumento de evaluación aplicado consta de un total de 22 preguntas abiertas y cerradas, y su elaboración se basó en el IRIS-Q (Interests \& Recruitment in Science questionnaire), el cual pregunta sobre algunas características educativas contextuales, interpersonales y experiencias personales.

La aplicación de los cuestionarios se llevó a cabo a los estudiantes de cuarto a noveno semestre en las carreras de STEM. Se recolectaron las direcciones electrónicas mediante la solicitud de apoyo de los responsables del aula de clases en universidades, formando con ello una base de datos para aplicar la encuesta de esta investigación. La aplicación de la encuesta se llevó a cabo durante el periodo comprendido de marzo a junio del año 2017. El total de los estudiantes encuestados fue de 1,154, donde la participación por institución se resume de la siguiente manera: para la Universidad Autónoma de Baja California (UABC) el 43.3\%, con el $30.5 \%$ la Universidad Politécnica de Baja California (UPBC) y con el 26,2\% la Universidad Estatal de Sonora (UES).

Es importante señalar que estos resultados serán punto de partida para la interpretación y análisis de datos dependerán de la matricula participante al momento de la encuesta. En la tabla 2 se muestra el desglose de la participación de cada institución involucrada en el estudio.

\section{Procesamiento de datos}

Una vez aplicados los cuestionarios a los estudiantes de las diferentes universidades, se obtuvieron el total de las encuestas ya contestadas a través del uso de una plataforma confiable de control "web-quest". Los resultados fueron revisados por el responsable del proyecto de investigación, para autorizar la captura de la información y procesamiento de la base de datos diseñada en formato Excel.
Posteriormente se utilizó el programa estadístico SPSS, para la extracción de los datos, mismos que se presentan e interpretan mediante tablas en el apartado de resultados. Así mismo, para la descripción de los datos se consideraron los siguientes criterios para su valoración: No importante, Poco importante, Neutral, Importante y Muy importante.

\section{Resultados de los datos de identificación del alumno}

Para la presente investigación se contó con el apoyo de estudiantes que se encuentran cursando un programa a nivel licenciatura en tres distintas universidades. Fueron recabadas un total de 1154 encuestas, donde la mayor participación se obtuvo de alumnos de género masculino con 947 encuestas mismas que representan el $82.1 \%$ del total, mientras que el resto, 207 encuestas que representan el $17.9 \%$ fueron de estudiantes de género femenino, como es posible observar en la Gráfica 1 de este reporte.

\begin{tabular}{|lrr|r|r|}
\hline Institución & $\begin{array}{c}\text { Matrícula } \\
\text { al } \\
\text { momento } \\
\text { del } \\
\text { estudio }\end{array}$ & $\begin{array}{c}\text { Matrícula } \\
\text { en } \\
\text { Ingeniería }\end{array}$ & $\begin{array}{c}\text { Estudiantes } \\
\text { participantes } \\
\text { en STEM }\end{array}$ & $\begin{array}{c}\text { Porcentaje } \\
\text { de } \\
\text { participación } \\
(\%)\end{array}$ \\
\hline UABC & 65,323 & $4,314 *$ & 500 & $43.3 \%$ \\
\hline UPBC & 1,472 & 1,342 & 352 & 30.5 \\
\hline UES & 2,021 & 408 & 302 & 26.2 \\
\hline Total & 68,816 & 6,064 & 1154 & $100 \%$ \\
\hline
\end{tabular}

Tabla 2 Población objeto de estudio del periodo 2017-1 Fuente: Directa. *Matrícula correspondiente solo a la Facultad de Ingeniería Mexicali-UABC

\section{Experiencia escolar para la elección de la carrera}

El aspecto con mayor puntuación manifestado por los estudiantes fue el relacionado con el interés por los temas científicos, donde el sexo femenino considera que es un aspecto muy importante con un $50.2 \%$ y el sexo masculino con un $45.0 \%$. La experiencia menos importante con $29.5 \%$ del sexo femenino y $29 \%$ masculino es la referente; experimentos y trabajo de laboratorio.

Evidentemente la práctica de laboratorio es indispensable para encarar los modelos STEM. Para ello, es preciso proponer la realización de prácticas desde temprana edad, no sólo para estudiantes sino también para docentes que no han tenido la oportunidad de desarrollar este tipo de habilidades (Bosh ,2011: 131-140). 


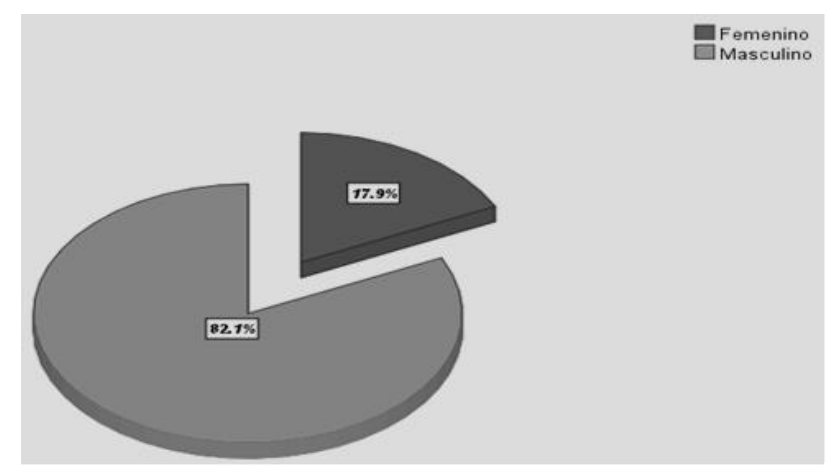

Grafica 1 Estudiantes por género

Fuente: Elaboración Propia

\section{Importancia que fueron las personas en la elección de la Carrera}

Es importante reconocer la influencia que ejercen las relaciones interpersonales ya sean de tipo familiar, profesores, entre otros, para que el estudiante decida seleccionar una determinada carrera. En lo que respecta a la madre o madrasta, el $37.7 \%$ de las mujeres mencionan que es muy importante y con el $30.2 \%$ de los hombres opinan lo mismo. Otra de las personas importantes en la selección de su carrera es el padre o padrastro con un $32.4 \%$ las mujeres opinan que es muy importante y con un $31.2 \%$ los hombres, y los menos importantes considerados por hombres y mujeres son los orientadores vocacionales y profesores en la escuela.

En la entrevista las mujeres expresaron tener apoyo por parte de sus padres para estudiar este tipo de carrera. Por lo anterior, se observa que la familia está cambiando su idea del rol tradicional de la mujer, a quien aconseja y apoya de manera decidida (Razo, 2008)

Es importante mencionar que, con el objetivo de fortalecer la imagen de padres de familia, el nuevo modelo educativo de la SEP establece como énfasis en el desarrollo infantil temprano, el promover programas de capacitación educativos para que los padres y madres de familia participen de manera más activa en el desarrollo de sus hijos.

\section{Actividades extraescolares importantes en la elección de la carrera}

Otro tema importante para la investigación además de conocer sobre el tipo de relaciones que generan mayor influencia en el alumno al momento de la elección de su carrera, es indagar sobre la relevancia que se les asigna a ciertos aspectos para que un alumno se incline por determinado programa universitario. En el caso de los libros y las revistas de divulgación científica, el $37.5 \%$ de las mujeres consideran que son importantes, mientras que el $30.2 \%$ de los hombres consideró que la opción importante es la mejor.

Por su parte, los libros o películas de ciencia ficción pueden resultar de gran motivación para que algunos estudiantes encuentren interés por determinados temas, sin embargo, la investigación realizada arrojó los siguientes resultados: la mayoría de los encuestados optó por la opción Importante con el $25.7 \%$ en el caso de las mujeres y un $29 \%$ que corresponden a los hombres opinan que es importante.

En esta categoría se incluyen las experiencias de aprendizaje que se desarrollan fuera del horario lectivo y de forma no dependiente del sistema formal, aunque sean actividades que se desarrollen en las instalaciones escolares o se dediquen al refuerzo de competencias STEM (por ejemplo, en colectivos más vulnerables).

\section{Opinión sobre si su género esta sobre representado en la carrera que estudia}

Se les cuestionó a los estudiantes si la carrera que estudiaban estaba sobre representada en su institución. En resumen, el porcentaje de opiniones muestra en la sumatoria de los géneros que el $61.6 \% \%$ de hombres y mujeres no piensan que su género está sobre representado, a diferencia del $38.4 \%$ que afirma estarlo.

En las preguntas abiertas los hombres expresaron que las mujeres son iguales de capaces que los hombres en las áreas de las ciencias, mientas que las mujeres consideran la necesidad de implementar un programa en sus instrucciones de modelos a seguir de mujeres exitosas para motivarlas en las áreas STEM. 
En el reporte del Primer Encuentro Nacional de Jóvenes en la Ingeniería, en noviembre de 2015 (Academia de Ingeniería México, 2015) en relación con la presencia de las mujeres en ingeniería, se señaló que todavía hay discriminación de género basada en tabúes como el hecho de la incapacidad intelectual de las mujeres ante la mayor presencia masculina, y que ello limita las oportunidades en el mercado laboral.

\section{Opinión sobre aspectos de aprendizaje y motivación a la carrera}

Adicionalmente, se consideró la opinión de los estudiantes sobre aspectos de aprendizaje y motivación a la carrera mediante cinco aspectos a valorar, mismos que son descritos a continuación:

- Lo hare mejor que el promedio en la carrera.

Aprendo fácilmente los temas de la carrera

Estoy seguro que soy bastante bueno en las asignaturas de la carrera

Está muy motivado para estudiar esta carrera

Probablemente voy a abandonar la carrera antes de que termine

La percepción de los estudiantes acerca del aprendizaje y la motivación son positivas. La más importante es la de no abandonar la carrera antes de que termine, seguida de aprendo fácilmente los temas de la carrera, lo cual es muy relevante ya que se encuentra en la etapa terminal de sus estudios.

\section{Opinión sobre la importancia del factor futuro}

Todos los porcentajes de los reactivos referentes a las prioridades del futuro tuvieron un porcentaje alto, como se indican a continuación; desarrollarse como persona, hacer algo de su interés, usar sus talentos y habilidades, trabajar en algo importante para la sociedad. Con un puntaje un poco más bajo corresponde a ganar dinero pronto.

\section{Habilidades que deben dominar los ingenieros}

Respecto a las preguntas abiertas acerca de la importancia del futuro en la ingeniería la mayoría expresó estar consciente de la creciente necesidad de ingenieros capacitados en habilidades creativas para generar innovación y patentes; interpretación de datos. Por lo tanto, en las habilidades blandas se refirieron a aspectos como liderazgo, ética profesional, el dominio del idioma inglés y el trabajo en equipo.

\section{Modelo de ecosistema CTS+I propuesto para implementación}

La complejidad de los nuevos requerimientos para los ingenieros del siglo XXI, debe dar paso a una nueva pedagogía, así como a la formación de un ecosistema formado por la industria, el gobierno, los diferentes sistemas educativos y la sociedad. Por ello, se propone un modelo al que llamaremos Ecosistema CTS+I, que englobe a la ciencia, la tecnología y la sociedad en un sistema innovador.

A partir del modelo propuesto, se logra la alineación de los retos actuales y futuros de la industria con los programas y esquemas educativos para la formación y desarrollo de talento y del mismo modo los programas del gobierno federal a través del nuevo modelo educativo.

Como prueba de campo y con el objetivo de validar el modelo, fue implementado en una de las empresas más dinámicas, tecnológicas, competitivas y de mayor crecimiento del Estado de Baja California, México. Lo cual nos permite además, observar la ruta de involucramiento de los actores en formación bajo un esquema STEM desde los niveles básicos hasta el profesional. Así como también, la manera en como los sistemas educativos se pueden incorporar al ecosistema, incluyendo las ventajas que el nuevo modelo mexicano de educación puede aportar desde un punto de enfoque global en el que participan ciudadanos corporativos.

El Ecosistema modelo se conforma a su vez de cuatro sistemas: La vinculación estratégica, el desarrollo económico y social, la competitividad y gestión y la inteligencia de negocios, como se muestra en la Figura 4. 
El sistema denominado Vinculación Estratégica, tiene como objetivo alinear las competencias técnicas y de ingeniería con los retos actuales y futuros, actualizando continuamente los programas de estudio a través de la ejecución de un mapa de ruta tecnológico, donde los académicos integraran nuevas competencias que formaran al estudiante en las tecnologías de punta y podrá experimentar y aplicarlo a través de los laboratorios compartidos entre las empresas y las institución educativas, asegurando así la infraestructura y programas educativos requeridos para la formación de talento.

Por otro lado, el cuerpo académico responsable de los módulos académicos, actualizan sus líneas de investigación a los requerimientos del entorno, impulsando la investigación aplicada y generando economía del conocimiento, a través de la incubación de proyectos, productos y servicios que beneficien a las empresas y a la sociedad.

El sistema denominado Competitividad y Gestión, tiene como objetivo impulsar la Ciencia, Tecnología e Innovación en la industria a través de los sistemas de gestión, incrementando los indicadores de competitividad de las empresas.

Sin embargo, además de la innovación que representa un gran beneficio para la empresa, parte fundamental es la integración de la sociedad a la industria, debido a que no solamente se requieren competencias técnicas o de ingeniería para desempeñar las funciones en la industria, sino que temas o módulos de gestión o "soft", tales como: trabajo en equipo, manejo de conflictos, DISC, inteligencia emocional, liderazgo, administración del cambio, entre otros.

Estrategias que permitirán al nuevo asociado de la empresa, tomar decisiones más rápidas, precisas, objetivas y con mejores resultados.

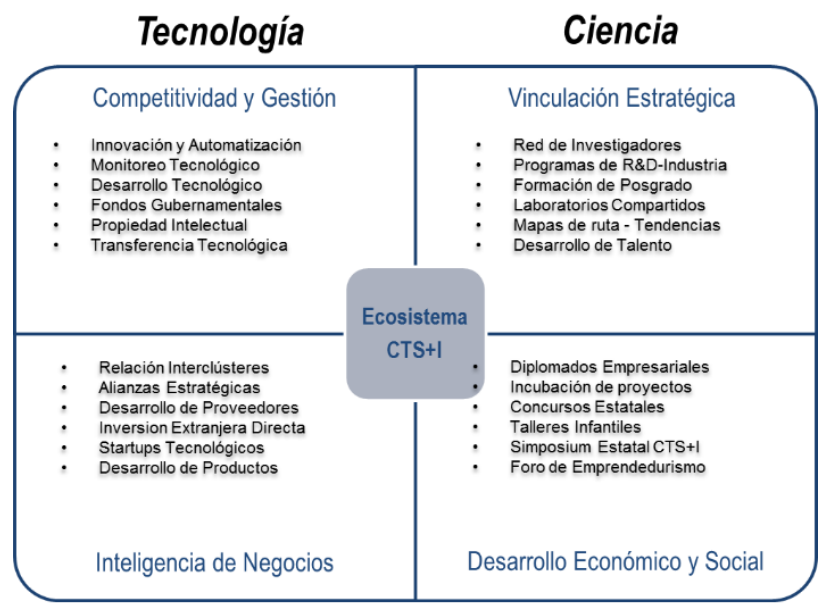

Innovación

Sociedad

Figura 4 Ecosistema CTS+I

Fuente: Elaboración Propia

Actualmente, se encuentra en operación un programa Dual por competencias STEM para estudiantes de preparatoria y licenciatura del último año, integrados al $100 \%$ en la empresa, adquiriendo precisamente estas competencias de gestión, adicional con los módulos definidos en su programa de estudios con la gran diferencia de la aplicación del conocimiento dentro de las funciones de la empresa.

Además, estos estudiantes tendrán mayor visión de la industria al conocer los departamentos que la conforman, los impactos y las responsabilidades, también, tendrán una formación integral con los módulos de gestión y certificaciones adicionales de administración de proyectos, resolución de problemas, análisis de datos y del idioma inglés. Para las empresas que adoptan este programa, tendrán como beneficio la pre-selección de talento y la optimización de la curva de aprendizaje, debido a que el estudiante estará formándose en la función en un esquema DUAL.

El sistema denominado Inteligencia de Negocios, tiene como objetivo interrelacionar a las empresas, instituciones educativas, cámaras, instituciones gubernamentales, inversionistas y la sociedad, identificando retos y problemáticas en común, a través de los clústeres y redes en la parte industrial, donde se desarrollen equipos multidisciplinarios para la búsqueda y solución del problema; a través de la metodología o flujo de innovación definido en cada programa, se desarrollan los productos y se generan nuevas empresas de base tecnológica. 
Se desarrollan proveedores con nuevos productos o servicios y se generan productos de alto valor, generando tecnología propia, protección intelectual y mejorando la competitividad de las empresas por la generación de proveeduría nacional y la generación de empleos de alto valor para el país. Por último, el sistema denominado Desarrollo Económico y Social, tiene como objetivo impulsar la innovación y creatividad a todos los niveles de la sociedad, con la finalidad de que se puedan generar proyectos que permitan mejorar la calidad de vida de la sociedad.

Para ello es importante que los actores de los diferentes programas cuenten con un enfoque interdisciplinario, donde las diversas disciplinas como la pedagogía, arquitectura, ingeniería y escuela de artes, permita detonar la creatividad y la innovación permeen a todos los niveles, desde la integración de la ciencia, tecnología, ingeniería, matemáticas y artes en los niños con talleres, juegos y retos que permitan despertar la creatividad hasta los concursos de innovación con retos definidos de acuerdo a las áreas estratégicas de los Estados y que atiendan o que generen una necesidad.

Así mismo, los talleres de innovación o gestión de tecnología para las empresas, han sido de gran utilidad para que puedan implantarlo en las empresas y crear sistemas que mejoren los indicadores de las empresas, y por otro lado, con este sistema estamos contribuyendo con el Plan Nacional de Desarrollo que busca impulsar las PYMEs y la innovación en nuestro país.

\section{Conclusiones}

Este documento presenta los resultados del análisis que se realizó a los estudiantes que actualmente cursan una carrera universitaria que sustenta la importancia de promover un modelo para la educación STEM.

Se observa que la brecha de genero todavía es un reto para la educación STEM en México, de las 1154 encuestas, el $82.1 \%$ corresponden al sexo masculino, mientras que el $17.9 \%$ fueron de estudiantes de género femenino.

En cuanto al indicador que mide la experiencia escolar para la elección de la carrera, el aspecto más importante su interés por los temas científicos y la referente experimentos y trabajo de laboratorio.
De acuerdo a (Bosh,2011: 131-140), los laboratorios ofrecen a los alumnos un poder sin precedentes para explorar, medir y aprender acerca del mundo de las ciencias. Eso es lo que se necesita para resolver los problemas que presenta la Sociedad: formar alumnos que puedan aprender acerca del mundo de las ciencias.

Respecto a la importancia de personas en la elección de la carrera tanto en hombres como mujeres, las más influyentes son la familia y los menos importantes considerados son los orientadores vocacionales y profesores en la escuela, por lo que reviste la importancia de la sensibilización de las instituciones en desarrollar un programa STEM para fortalecer las habilidades del ingeniero en el siglo XXI. En el reporte de la Academia de Ingeniería de México refiere la eficiencia formativa de los ingenieros, de otra manera su deficiencia genera costosas actividades de remediación o por retraso formativo, así la deserción y frustración de los jóvenes. México no puede aspirar a acceder a un mayor nivel de desarrollo si la cadena formativa completa desde la educación básica hasta el posgrado no satisface las exigencias de calidad y cantidad que requiere el país.

Con este objetivo, el nuevo modelo educativo de la SEP establece como énfasis en el desarrollo infantil temprano, el promover programas de capacitación y material didáctico actualizado para los profesores de todos los niveles educativos.

En lo que respecta a las actividades extraescolares que motivan más a los alumnos a indagar sobre la relevancia de la ciencia, se encuentran libros y revistas de divulgación científica, ferias y clubes de ciencia y la fotografía científica.

En lo referente a la sobre representación de género, aceptaron que si existe. En las preguntas abiertas los hombres expresaron que las mujeres tienen la misma capacidad que los hombres en las áreas de las ciencias, al mismo tiempo que consideran la necesidad de implementar un programa en sus instrucciones de modelos a seguir de mujeres exitosas para motivarlas en las áreas STEM. 
Adicionalmente, se consideró la opinión de los estudiantes sobre aspectos de aprendizaje y motivación a la carrera. La percepción de los estudiantes acerca del aprendizaje y la motivación son positivas. La más importante es la de no abandonar la carrera antes de que termine, seguida de aprendo fácilmente los temas de la carrera, lo cual es muy relevante ya que se encuentra en la etapa terminal de sus estudios.

Los reactivos valorados con los porcentajes más altos son las opiniones sobre la importancia del factor futuro, lo que indica un perfil optimista y están relacionadas con; desarrollarse como persona, hacer algo de su interés, usar sus talentos y habilidades, así como el trabajar en algo importante para la sociedad. Con un puntaje un poco más bajo se ubicó el concepto de ganar dinero pronto. También con puntos muy positivos eligieron habilidades blandas como son liderazgo, ética profesional, inglés entre otras En general, los futuros ingenieros le asignan un alto valor de utilidad a las carreras STEM.

La implementación de un sistema educativo basado en STEM+A es un proceso difícil que requiere de la intervención de actores de todos los sectores de gobierno, educación, productivo y la sociedad. La educación científica y tecnológica debe al igual que todas las otras ramas del conocimiento estar complementada con la responsabilidad social y el enriquecimiento del bagaje cultural del estudiante para así responder a los requerimientos de la " 4 a revolución industrial". El diseño de ecosistemas operativos para la mejora de la producción que involucran las habilidades adquiridas en un modelo educativo STEM, es factible y necesario para el éxito futuro en la generación de riquezas y bienestar de nuestro México.

El ecosistema CTS+I diseñado a la medida de condiciones propias de nuestro país e implementado en una industria de alta tecnología ha sido validado y exitoso, y puede ser replicado y mejorado en otras organizaciones a lo largo y ancho de nuestro país.

\section{Referencias}

Academia de Ingeniería México (2015). Primer Encuentro Nacional de Jóvenes en Ingeniería. Recuperado de http://www.ai.org.mx/ai/index. php/9-uncategorised/546-primer-encuentro-nacional-de-jovenes-en-la-ingenieria

Archer, L., DeWitt, J., Osborne, J., Dillon, J., Willis, B., \& Wong, B. (2012). "Science aspirations, capital, and family habitus: How families shape children's engagement and identification with science". American Educational Research Journal, 49(5), 881-908

Bjørnstad, R., Fredriksen, D., Gjelsvik, M. L., \& Stølen, N.M. (2008). "Tilbud og etterspørsel etter. Rapporter" 2008/29, Statistisk sentralbyrå, Statistics Norway Oslo-Kongsvinger.

Blickenstaff, J. (2005). Women and science careers: leaky pipeline or gender. Gender and Education: Taylor \&Francis. Vol.17.

Boe, M. V., Henriksen, E. K., Lyons, T., \& Schreiner, C. (2011). Participation in science and technology: Young people's achievementrelated choices in late-modern societies. Studies in Science Education, 47(1), 37-72.

Bosch, Horacio E.; Di Blasi, Mario A.; Pelem, Mariano E.; Bergero, Mercedes S.; Carvajal, Leonor; Geromini, Noemí S.” Nuevo Paradigma Pedagógico para Enseñanza de Ciencias y matemáticas". Avances en Ciencias e Ingeniería, vol. 2, núm. 3, 2011, pp. 131-140, Executive Business School La Serena, Chile

Carvajal, Jaime (2017). "La Cuarta Revolución Industrial o Industria 4.0 y su Impacto en la Educación Superior en Ingeniería en Latinoamérica y el Caribe". 15th LACCEI International Multi-Conference for Engineering, Education, and Technology: "Global Partnerships for Development and Engineering Education”, 19-21 July 2017, Boca Dabney, K. P., Chakraverty, D. and TAI, R. H. (2013), The Association of Family Influence and Initial Interest in Science. Sci. Ed., 97: 395-409. doi: $10.1002 /$ sce. 21060 
Del Val José Luis (2016). Industria 4.0: la transformación digital de la industria. Conferencia de Directores y Decanos de Ingeniería Informática. Disponible en: http://coddii.org/wpcontent/uploads/2016/10/Informe-CODDIIIndustria-4.0.pdf

Escudero Nahón, Alexandro. (2018). Redefinición del "aprendizaje en red" en la cuarta revolución industrial. Apertura, 10 (1), pp. $149-163$.

http://dx.doi.org/10.18381/Ap.v10n1.1140

Jacob Clark Blickenstaff (2005) "Women and science careers: leaky pipeline or gender filter?", Gender and Education, 17:4, 369-386, DOI: 10.1080/09540250500145072. Taylor \& Francis.

Rascón Octavio, Academia Mexicana de ingeniería. Reporte El estado del arte y prospectiva de la ingeniería en México y en el mundo. Consultado en: http://www.ai.org.mx/sites/default/files/10.pros pectiva_de_la_ingenieria_en_mexico_y_en_el_ mundo.pdf. (2010)

OECD (2015), "The ABC of Gender Equality in Education": Aptitude, Behaviour, Confidence, PISA, OECD Publishing. http://dx.doi.org/10.1787/9789264229945-en. Organización de las naciones Unidas para la educación, la ciencia y la cultura (UNESCO) http://www.cromo.com.uy/unesco-el-mundonecesita-mas-ciencia-n583092

Razo, L. (2008). “La inserción de las mujeres en las carreas de ingeniera y tecnología". Perfiles Educativos, 30 (121).

Sanders, M. (2009). "STEM, STEM education, STEM mania”. Technology Teacher, December/January, 68 (4), 20-26.

Salas Daniel (2016). "Revolución 4.0". RIINN: Revista Ingeniería e Innovación. Vol. 4, Num. 2. http://revistas.unicordoba.edu.co/index.php/rii/a rticle/view/1174/1452

Scott,J, (2014) "Redistributive Impact and Efficiency of Mexico's Fiscal System", . Public Finance Review, Vol. 42, No. 3, pp. 368-390
Swain Ricardo (2017). Modelo Educativo para la Industria 4.0. Academia de Ingeniería México. Pp 17 -22

Toma, R. B. (2015). "Fundamento, desempeño e inconvenientes de la indagación escolar". [Trabajo Fin de Grado]. Burgos: Universidad de Burgos

Uthoff, Andras (2006), "Brechas del Estado de bienestar y reformas a los sistemas". Publicación de las Naciones Unidas 